\title{
Concurrent Radiosurgery and Systemic Therapies for Melanoma Brain Metastases: A Systematic Review
}

\author{
Bradley D. Weaver ${ }^{1}$, James R. Goodman ${ }^{2}$, Randy Jensen ${ }^{3}$ \\ 1. Oncology, University of Utah, Salt Lake City, USA 2. Anesthesiology, Oregon Health \& Science University, Portland, \\ USA 3. Neurosurgery, University of Utah, Salt Lake City, USA
}

Corresponding author: Randy Jensen, randy.jensen@hsc.utah.edu

\begin{abstract}
Intracranial metastatic melanoma is a major challenge for neuro-oncological teams. Historically, treatment has focused on surgical or radiosurgical treatment of appropriate lesions, mostly for palliative purposes. Immunotherapies and other targeted therapies (BRAF/mitogen-activated protein kinase kinase inhibitors (BRAFi/MEKi)) are mainstays of advanced melanoma therapy, yet the optimal timing and synergistic properties of concurrent combinations of these systemic therapies and stereotactic radiosurgery (SRS) are poorly understood. We performed a systematic review of the MEDLINE and Scopus databases focused on outcomes after therapy using SRS and either immunotherapies or targeted therapies in an effort to define the optimal timing. We defined concurrent therapy as SRS within three months of treatment with any systemic therapy. End points included local control, distant control, overall survival, and toxicities. We identified five retrospective cohort studies from the literature. These studies found that concurrent SRS plus immunotherapy or BRAFi/MEKi is well tolerated by most patients and generally improved local control, distant control, and overall survival. Importantly, no significant increases in toxicities were noted with concurrent therapy. Combining concurrent SRS with immunotherapy or BRAFi/MEKi may offer important advances for patients with intracranial metastatic melanoma. To address interstudy heterogeneity, we propose reporting two major time intervals defining "concurrent treatment": concurrent-SRS ( $\leqslant 4$ weeks) and peri-SRS ( $\leqslant 3$ months). Future large-scale, prospective trials considering truly concurrent SRS therapies with systemic therapies are desperately needed.
\end{abstract}

Received 11/04/2019 Review began 11/06/2019 Review ended 11/11/2019 Published 11/13/2019

๑) Copyright 2019 Weaver et al. This is an open access article distributed under the terms of the Creative Commons Attribution License CC-BY 3.0., which permits unrestricted use, distribution, and reproduction in any medium, provided the original author and source are credited.
Categories: Radiation Oncology, Neurosurgery, Oncology

Keywords: melanoma, stereotactic radiosurgery, targeted therapy, immunotherapy, brain metastases

\section{Introduction And Background}

Intracranial metastatic melanoma is a devastating and common occurrence in patients with advanced melanoma. As of 2011 , more than $40 \%$ of patients with metastatic melanoma experienced brain metastasis, and this number is increasing [1-2]. Stereotactic radiosurgery (SRS) is a safe and effective modality for treating many types of primary and metastatic brain tumors and is commonly used for the treatment of melanoma brain metastasis. BRAF-V600E is the most common activating mutation found in melanoma. After the molecular diagnosis is established, targeted therapies (TTs) such as BRAF/mitogen-activated protein (MAP) kinase kinase (MEK) inhibitors (BRAFi/MEKi), which block the activated MAP-kinase cascade are employed [3]. Immunotherapeutics (IMTs) such as ipilimumab (anti-CTLA4 [cytotoxic Tlymphocyte associated protein 4] therapy) have yielded improved overall survival from metastatic melanoma (two large, phase III trials), and along with nivolumab/pembrolizumab (anti-PD1 [programmed cell death protein 1] therapy), comprise the cornerstone of current melanoma immunotherapy [4-5]. Recently, phase II trials have begun investigating the effectiveness of IMTs with and without SRS [NCT02085070; NCT02374242; NCT02460068; NCT02320058]. Importantly, the optimal timing of combination systemic therapy and SRS is yet to be defined, particularly for IMTs, and preclinical studies suggest that concurrent therapy may be superior to staggered drug and SRS administration [6]. We undertook a systematic review of studies involving a window of concurrent systemic therapy within three months of SRS treatment, defined as administration of IMT or TT within three months of SRS, in an effort to better define the optimal timing.

\section{Review}

\section{Database review}

Two separate reviewers performed Preferred Reporting Items for Systematic Reviews and Meta-analyses (PRISMA)-based systematic reviews of both Scopus and MEDLINE databases (October 2018) using "stereotactic radiosurgery" and "melanoma" as keywords. Articles were included if they examined the treatment of intracranial metastatic melanoma with SRS and BRAFi/MEKi inhibitors or immunotherapeutic checkpoint inhibitors (i.e., anti-PD1 and anti-CTLA4 monoclonal antibodies). Studies were included if they reported $\leqslant 30 \%$ of patients previously, or concurrently, treated with whole-brain radiotherapy. Critically, studies were included only if they described concurrent combinations of SRS and systemic therapies (i.e., 
systemic therapy within a three-month window before or after SRS treatment) (Table 1). Endpoints of interest included overall survival, local control, distant control, and treatment toxicities.

After a systematic review of the literature, five articles were included for qualitative analysis (Figure 1). Hazard ratios (HRs) reported were transformed from failure to control, if necessary, using $1 / \mathrm{HR}$. If a study presented both univariate and multivariate analyses of an outcome measure, the multivariate analysis was included in our qualitative assessment.

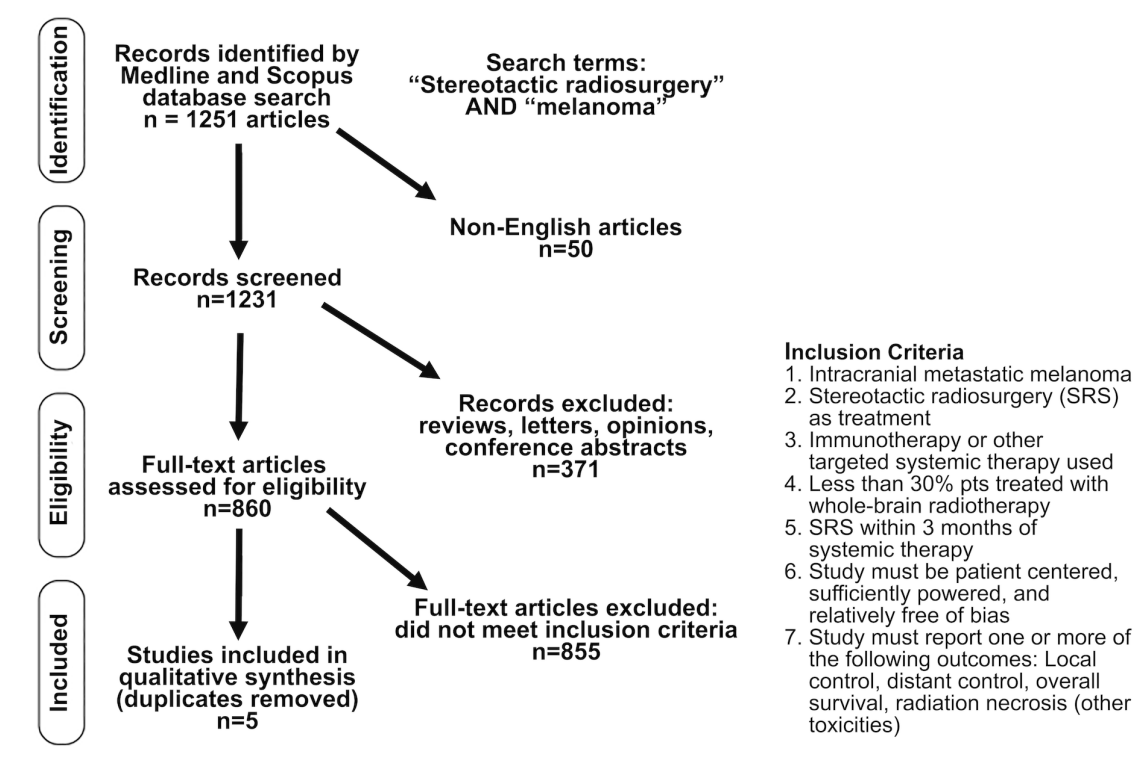

FIGURE 1: Diagram of PRISMA workflow representing search strategy, results, and inclusion criteria

\section{Studies identified}

The five retrospective cohort studies that met our inclusion criteria all provided only low-quality evidence with heterogeneous outcomes (Table 1; GRADE (Grading of Recommendations Assessment, Development, and Evaluation) criteria); therefore, a meta-analysis of the data was not possible [7-11]. A major source of heterogeneity in all studies of SRS and systemic therapies is the definition of "concurrent" therapy. To address this issue, we propose assessment during two key intervals: a four-week interval (concurrent therapy) and a three-month window (peri-SRS therapy). 


\section{Cureus}

\begin{tabular}{|c|c|c|c|c|c|c|c|c|c|}
\hline Article & $\begin{array}{l}\text { Number } \\
\text { of } \\
\text { patients }\end{array}$ & $\begin{array}{l}\text { Total } \\
\text { no. } \\
\text { of } \\
\text { brain } \\
\text { mets }\end{array}$ & $\begin{array}{l}\text { Type of } \\
\text { radiosurgery }\end{array}$ & $\begin{array}{l}\text { Type(s) of targeted and } \\
\text { immunotherapies }\end{array}$ & $\begin{array}{l}\text { Concurrent } \\
\text { treatment } \\
\text { definition }\end{array}$ & $\begin{array}{l}\text { Patients } \\
\text { (\% of } \\
\text { total) } \\
\text { who had } \\
\text { WBRT }\end{array}$ & Endpoints measured & Statistics used & $\begin{array}{l}\text { GRADE quality } \\
\text { and bias } \\
\text { assessment }\end{array}$ \\
\hline $\begin{array}{l}\text { Acharya } \\
\text { et al. } \\
\text { (2017) }\end{array}$ & 72 & 233 & $\begin{array}{l}\text { Single-fraction } \\
\text { SRS Leksell } \\
\text { Gamma Knife }\end{array}$ & $\begin{array}{l}\text { Anti-PD1/anti-CTLA4 = } \\
\text { nivolumab/ pembrolizumab, } \\
\text { ipilimumab; BRAFi/MEKi = } \\
\text { dabrafenib/ trametinib, } \\
\text { vemurafenib }\end{array}$ & 3 months & 9.7 & $\begin{array}{l}\text { Distant brain failure, } \\
\text { local failure, overall } \\
\text { survival, neurotoxicity }\end{array}$ & $\begin{array}{l}\text { Fisher's exact test and Wilcoxon } \\
\text { rank-sum; Kaplan Meier and Cox } \\
\text { proportional hazards regression } \\
\text { model for hazard ratios }\end{array}$ & $\begin{array}{l}\text { LOW: small, } \\
\text { retrospective } \\
\text { cohort study. } \\
\text { No downgrade } \\
\text { required. }\end{array}$ \\
\hline $\begin{array}{l}\text { Ahmed } \\
\text { et al. } \\
(2016)\end{array}$ & 96 & 314 & $\begin{array}{l}\text { Single-fraction } \\
\text { BrainLab Novalis } \\
\text { Classic LINAC }\end{array}$ & $\begin{array}{l}\text { Anti-PD1/anti-CTLA4 = } \\
\text { nivolumab/ pembrolizumab, } \\
\text { ipilimumab; BRAFi/MEKi = } \\
\text { dabrafenib/ trametinib, } \\
\text { vemurafenib }\end{array}$ & $\begin{array}{l}3 \text { months; BRAF/ } \\
\text { MEK inhibitors } \\
\text { held for 2-3 } \\
\text { days } \\
\text { before/after SRS }\end{array}$ & $\begin{array}{l}\text { Not } \\
\text { reported }\end{array}$ & $\begin{array}{l}\text { Distant brain control, } \\
\text { local control, } \\
\text { progression-free } \\
\text { survival, overall } \\
\text { survival, neurotoxicity }\end{array}$ & $\begin{array}{l}\text { Kruskal-Wallis, Pearson's Chi- } \\
\text { squared, Fisher's exact tests. } \\
\text { Kaplan Meier and log-rank tests. } \\
\text { Cox prop hazards for hazard } \\
\text { ratios. }\end{array}$ & $\begin{array}{l}\text { LOW: small, } \\
\text { retrospective } \\
\text { cohort study. } \\
\text { No downgrade } \\
\text { required. }\end{array}$ \\
\hline $\begin{array}{l}\text { Diao et } \\
\text { al. } \\
(2018)\end{array}$ & 72 & 310 & $\begin{array}{l}\text { Single-fraction } \\
\text { SRS Elekta } \\
\text { Perfexion Gamma } \\
\text { Knife(s) }\end{array}$ & Anti-CTLA4 = ipilimumab & weeks & 8.3 & $\begin{array}{l}\text { Local failure, } \\
\text { treatment-related } \\
\text { imaging changes, } \\
\text { tumor, and edema } \\
\text { volumes, neurotoxicity }\end{array}$ & $\begin{array}{l}\text { Kruskal-Wallis, Pearson's Chi- } \\
\text { squared, Fisher's exact tests. } \\
\text { Kaplan Meier and Cox } \\
\text { proportional hazards for hazard } \\
\text { ratios. }\end{array}$ & $\begin{array}{l}\text { LOW: small, } \\
\text { retrospective } \\
\text { cohort study. } \\
\text { No downgrade } \\
\text { required. }\end{array}$ \\
\hline $\begin{array}{l}\text { Diao et } \\
\text { al. } \\
\text { (2018) }\end{array}$ & 91 & 256 & $\begin{array}{l}\text { SRS Perfexion } \\
\text { Gamma Knife }\end{array}$ & Anti-CTLA4 = ipilimumab & $\begin{array}{l}4 \text { weeks (peri = } 4 \\
\text { wk-3 mo) }\end{array}$ & 7.6 & $\begin{array}{l}\text { Distant brain failure, } \\
\text { local failure, failure-free } \\
\text { survival, overall } \\
\text { survival, neurotoxicity }\end{array}$ & $\begin{array}{l}\text { Kruskal-Wallis, Pearson's Chi- } \\
\text { squared, Fisher's exact tests. } \\
\text { Kaplan Meier and Cox } \\
\text { proportional hazards for hazard } \\
\text { ratios. }\end{array}$ & $\begin{array}{l}\text { LOW: small, } \\
\text { retrospective } \\
\text { cohort study. } \\
\text { No downgrade } \\
\text { required. }\end{array}$ \\
\hline $\begin{array}{l}\text { Yusuf et } \\
\text { al. } \\
\text { (2017) }\end{array}$ & 51 & 167 & $\begin{array}{l}\text { CyberKnife/Varian } \\
\text { Trilogy LINAC }\end{array}$ & $\begin{array}{l}\text { Anti-PD1/anti-CTLA4 = } \\
\text { ipilimumab/ pembrolizumab }\end{array}$ & $\begin{array}{l}4 \text { weeks (peri = } 4 \\
\text { wk-3 mo) }\end{array}$ & 5.8 & $\begin{array}{l}\text { Distant brain failure, } \\
\text { local failure, percent } \\
\text { lesion regression, } \\
\text { overall survival, } \\
\text { neurotoxicity }\end{array}$ & $\begin{array}{l}\text { Kruskal-Wallis, Pearson's Chi- } \\
\text { squared, Fisher's exact tests. } \\
\text { Kaplan Meier and Cox } \\
\text { proportional hazards for hazard } \\
\text { ratios. }\end{array}$ & $\begin{array}{l}\text { LOW: small, } \\
\text { retrospective } \\
\text { cohort study. } \\
\text { No downgrade } \\
\text { required. }\end{array}$ \\
\hline
\end{tabular}

\section{TABLE 1: Details of the retrospective cohort studies included in this review}

WBRT, whole-brain radiation therapy; SRS, stereotactic radiosurgery; PD1, programmed cell death protein 1; CTLA4, cytotoxic T-lymphocyteassociated protein 4; LINAC, linear accelerator; BRAFi, BRAF inhibitor; MEKi, MEK inhibitor

Results of Concurrent Immunotherapy and SRS

Concurrent therapy was generally favored for all reported outcomes. Possibly, the largest effect was on distant brain control combining IMT+SRS (HRs $\geqslant 2$ for distant control in all but one case; Figure 2). Local control favored concurrent therapy, but this trend was obscured by unexpected differences in HRs for similar patient populations (Figure 2) [9,11]. Overall survival with concurrent IMT+SRS was also improved. Only Acharya et al. found a statistically insignificant decrease in HR for overall survival in patients receiving concurrent IMT and SRS (Figure 2) [7]. There was no significant difference in toxicities between the SRS only and the SRS + IMT treatment groups (Table 2). 


\section{Cureus}

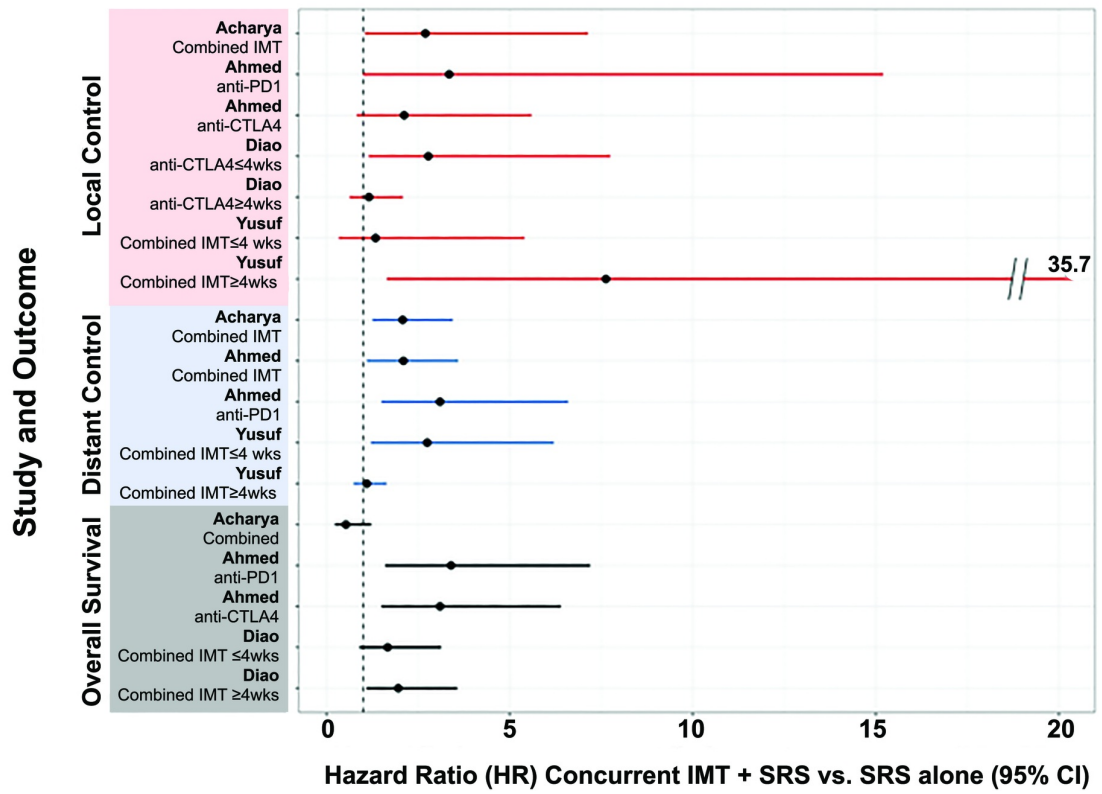

FIGURE 2: Efficacy of concurrent immunotherapy and SRS for melanoma brain metastases

IMT, immunotherapy; CTLA4, cytotoxic T-lymphocyte-associated protein 4; PD1, programmed cell death protein 1; wks, weeks; SRS, stereotactic radiosurgery 


\section{Cureus}

\begin{tabular}{|c|c|c|c|c|c|}
\hline Study & Overall Survival & Local Control & Distant Control & Toxicities & Comparator \\
\hline $\begin{array}{l}\text { Acharya } \\
\text { et al. } \\
\text { (2017) }\end{array}$ & $0.53(0.23-1.22) p=N S$ & $2.70(1.05-7.14) p=0.04^{*}$ & $\begin{array}{l}2.08(1.25-3.45) \\
\mathrm{p}=0.003^{\star}\end{array}$ & $\begin{array}{l}\text { Toxicities were } \\
\text { noted in all } \\
\text { groups; no } \\
\text { differences }\end{array}$ & $\begin{array}{l}\text { SRS alone; } \\
\text { controlled for \# } \\
\text { MBM and steroid } \\
\text { use }\end{array}$ \\
\hline $\begin{array}{l}\text { Ahmed } \\
\text { et al. } \\
\text { (2016) }\end{array}$ & $\begin{array}{l}\text { Anti-PD-1 } 3.4(1.6-7.2) p= \\
0.0009 ; \text { Anti-CTLA4 } 3.1 \\
(1.5-6.4) p=0.002^{* *}\end{array}$ & $\begin{array}{l}\text { Anti-PD-1 } 3.35 \text { (0.99-15.2) } \\
\mathrm{p}=0.051 ; \text { Anti-CTLA4 } 2.12 \\
(0.82-5.6) \mathrm{p}=0.12 \text { ** }\end{array}$ & $\begin{array}{l}\text { Anti-PD1 3.1 (1.5-6.6) } \\
\mathrm{p}=0.001 \text {; combined IMT } \\
2.1(1.1-3.6) \mathrm{p}=0.02 \text { ** }\end{array}$ & $\begin{array}{l}\text { Toxicities were } \\
\text { noted in all } \\
\text { groups; no } \\
\text { differences }\end{array}$ & $\begin{array}{l}\text { SRS alone; } \\
\text { chemotherapy } \\
\text { controlled }\end{array}$ \\
\hline $\begin{array}{l}\text { Diao et } \\
\text { al. } \\
(2018)\end{array}$ & $\mathrm{NR}$ & $\begin{array}{l}\text { Timing } \leq 4 \text { weeks } 2.78 \\
(1.15-7.75) ;{ }^{*} \text { timing } \geq 4 \\
\text { weeks } 1.16(0.63-2.09)^{*}\end{array}$ & NR & $\begin{array}{l}\text { Any lesion } \\
\text { hemorrhage HR = } \\
2.13(0.987-4.72)^{*}\end{array}$ & $\begin{array}{l}\text { Anti-CTLA4 vs } \\
\text { SRS alone }\end{array}$ \\
\hline $\begin{array}{l}\text { Diao et } \\
\text { al. } \\
\text { (2018) }\end{array}$ & $\begin{array}{l}\text { Timing } \leq 4 \text { weeks } 1.67 \\
(0.90-3.13)^{\star} ; \text { timing } \geq 4 \\
\text { weeks } 1.96(1.09-3.57)^{\star} \\
p=0.02 ;\end{array}$ & $\mathrm{NR}$ & $\mathrm{NR}$ & $\begin{array}{l}\text { Toxicities were } \\
\text { noted in all } \\
\text { groups; no } \\
\text { differences }\end{array}$ & $\begin{array}{l}\text { Anti-CTLA4 vs } \\
\text { SRS alone }\end{array}$ \\
\hline $\begin{array}{l}\text { Yusuf } \\
\text { et al. } \\
\text { (2017) }\end{array}$ & $\begin{array}{l}\text { Median peri-SRS = } 7.4 \mathrm{mo} ; \\
\text { SRS alone = } 7.1 \mathrm{mo} \\
\mathrm{p}=0.212\end{array}$ & $\begin{array}{l}\text { Concurrent } 1.34(0.33- \\
5.41)^{\star} ; \text { peri } 7.63(1.64- \\
35.7)^{\star}\end{array}$ & $\begin{array}{l}\text { Concurrent } 2.75(1.21- \\
6.21)^{\star} ; \text { peri } 1.10(0.74- \\
1.64)^{\star}\end{array}$ & $\begin{array}{l}\text { Toxicities were } \\
\text { noted in all } \\
\text { groups; no } \\
\text { differences }\end{array}$ & SRS alone \\
\hline
\end{tabular}

\section{TABLE 2: Studies considering treatment efficacy of SRS and immunotherapy}

SRS, stereotactic radiosurgery; HR, Hazard ratio; NS; not significant; NR, not reported; MBM, melanoma brain metastases; PD-1, programmed cell death protein 1; CTLA4, cytotoxic T-lymphocyte-associated protein 4; IMT, immunotherapy

* Converted from failure to control; ** chemotherapy controlled; ${ }^{* * *}$ compared with immunotherapy before SRS

Regression or control of lesions outside of the initial radiation field, called the abscopal effect, may drive favorable outcomes for distant brain tumors in patients treated with concurrent IMT+SRS [12-14]. The abscopal effect is an immune system-mediated effect that requires T-cell effector function on tumorassociated neoantigens (TAAs) and immune-enhancing cytokine release in the tumor microenvironment. This may synergize with checkpoint inhibitors, releasing the brakes on antitumor immunity (Figure 3) [1517]. The reactivation of innate immune-sensing and interferon responses in tumor cells, which is critical for antitumor immunity, is attributed to the expression of endogenous retroviral sequences and other genomic 'dark matter' after epigenetic therapies [18,19-21]. Combining epigenetic, stereotactic radiosurgical, and immune-checkpoint therapies may hold great promise for patients with melanoma brain metastasis. 


\section{Cureus}

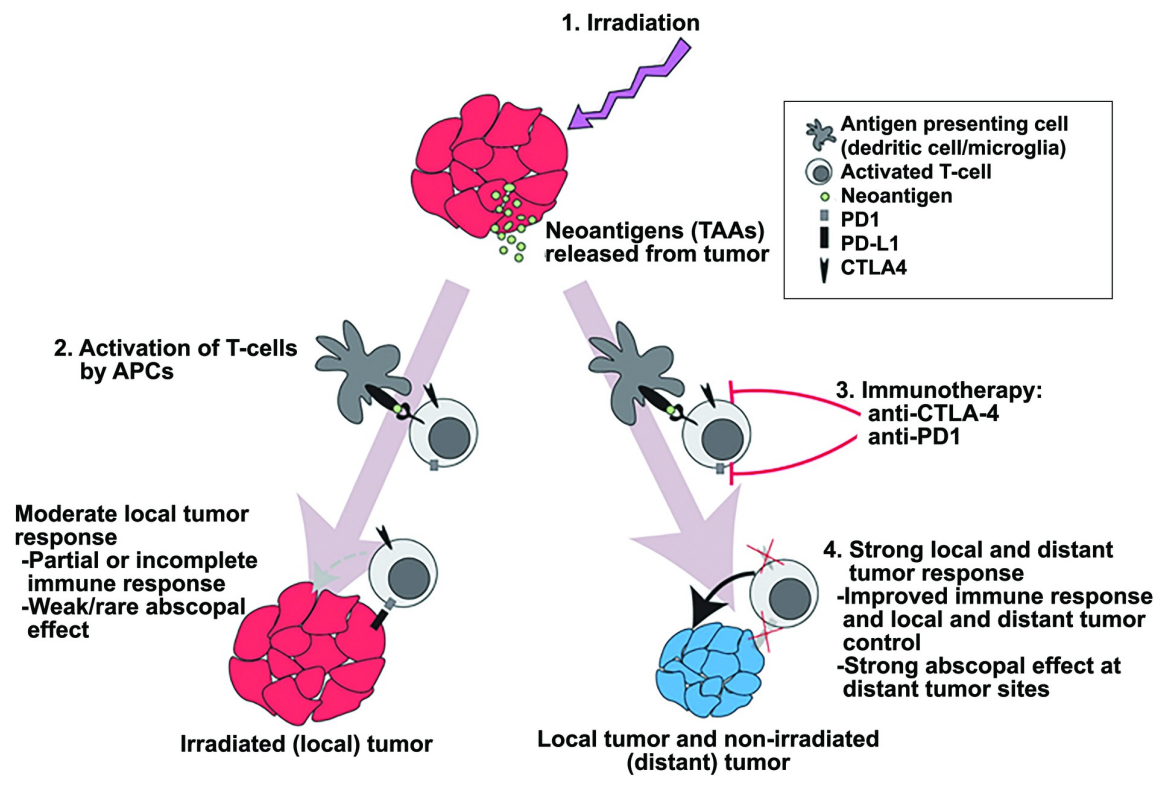

\section{FIGURE 3: Model of increased abscopal response in combination immunotherapy and radiosurgery}

1) Irradiation of tumor causes the release of TAAs. 2) Cytotoxic T-cell activation by APCs. 3) Immunotherapy facilitates T-cell activation (anti-CTLA4) and prevents immune checkpoint activation (anti-PD1). 4) Increased abscopal effect and local tumor responses.

TAA, tumor-associated neoantigen; APC, antigen-presenting cell; CTLA4, cytotoxic T-lymphocyte-associated protein 4; PD1, programmed cell death protein 1; PD-L1, programmed death-ligand 1; TT, targeted therapy; SRS, stereotactic radiosurgery; BRAFi, BRAF inhibitor; MEKi, MEK inhibitor. @ Department of Neurosurgery, University of Utah

Results of BRAFi/MEKi and Stereotactic Radiosurgery

Inhibitors of the MAP-kinase pathway have become a mainstay of melanoma treatment, particularly in the setting of activating BRAF mutations. Importantly, patients treated with targeted therapies after molecular diagnosis have significantly better progression-free and overall survival than patients treated with dacarbazine or placebo [22-25]. There is little information regarding the optimal timing of targeted therapy dosage relative to SRS, but many oncological teams withhold targeted therapies for 3-5 days surrounding SRS treatment. Studies that report the efficacy of SRS and targeted therapies are summarized in Table 4, including reason for exclusion from our systematic review [26-30]. Improved brain tumor control and overall survival evident in concurrent or post-SRS (Figure 4; Table 3) BRAFi/MEKi may reflect increased accessibility of these therapies to the brain tumor environment after SRS, yet concurrent vemurafenib or dabrafenib and SRS has been reported to increase risk for radiation necrosis and Grade $\geqslant 3$ adverse events, especially skin toxicities [30-32]. Of interest, there were no significant differences in toxicities in patients receiving concurrent BRAFi/MEKi+SRS versus SRS alone, indicating that a small pause directly surrounding SRS may be sufficient to ensure patient safety (Table 3). 


\section{Cureus}

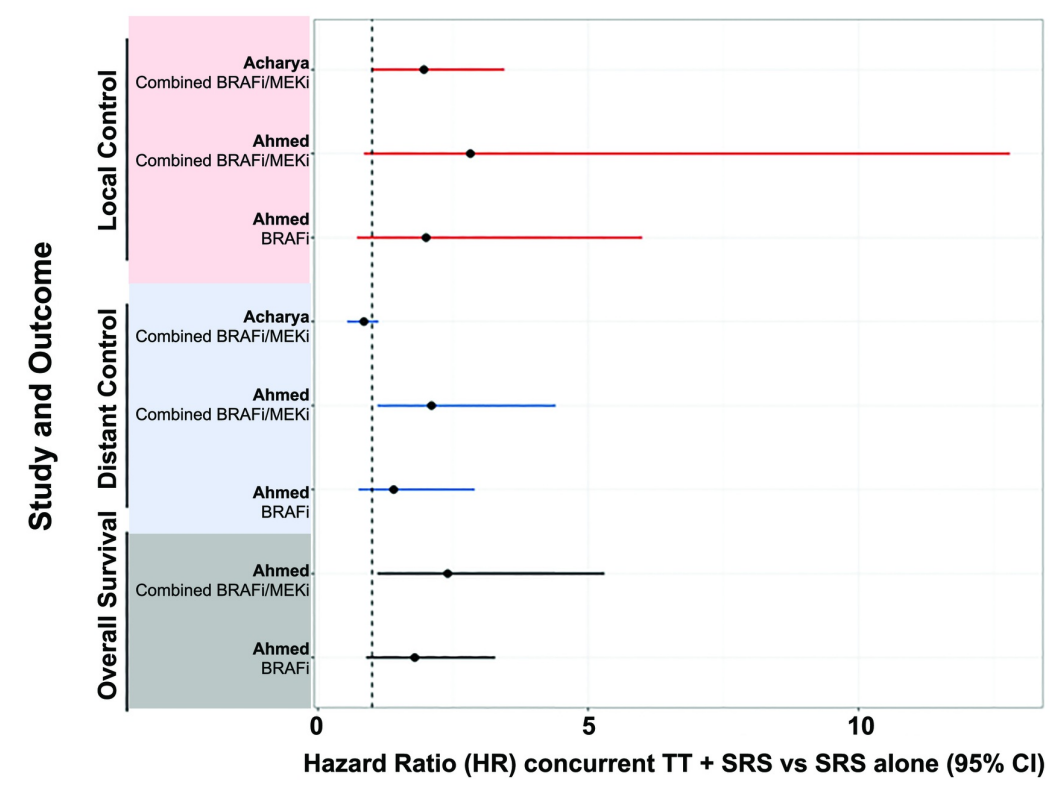

FIGURE 4: Efficacy of concurrent targeted therapy and SRS for melanoma brain metastases

BRAFi, BRAF inhibitor therapy; MEKi, MEK inhibitor therapy; TT, targeted therapy; SRS, stereotactic radiosurgery

\begin{tabular}{|c|c|c|c|c|c|}
\hline Study & Overall Survival & Local Control & Distant Control & Toxicities & Comparator \\
\hline $\begin{array}{l}\text { Acharya } \\
\text { et al. } \\
(2017)\end{array}$ & NR & $1.96(0.99-3.45)^{\star} p=0.054$ & $\begin{array}{l}0.85(0.54-1.12)^{\star} ; \\
\text { BRAFi/MEKi vs BRAFi alone } \\
p=0.011\end{array}$ & $\begin{array}{l}\text { Toxicities noted } \\
\text { in all group; no } \\
\text { differences }\end{array}$ & $\begin{array}{l}\text { SRS alone with } \\
\text { multiple } \\
\text { subgroup } \\
\text { analyses }\end{array}$ \\
\hline $\begin{array}{l}\text { Ahmed } \\
\text { et al. } \\
(2016)\end{array}$ & $\begin{array}{l}\text { BRAFi/MEKi } 2.4(1.1- \\
5.3) \mathrm{p}=0.02 ; \mathrm{BRAFi} \\
1.79(0.89-3.28) \text { ** }\end{array}$ & $\begin{array}{l}\text { BRAFi/MiEKi } 2.82(0.85- \\
12.8) p=0.09 ; \text { BRAFi } 2 \\
(0.72-6.0) p=0.18^{\star *}\end{array}$ & $\begin{array}{l}\text { BRAFi/MEKi } 2.1(1.1-4.4) p \\
=0.03 ; \text { BRAFi alone } 1.4 \\
(0.75-2.9) p=0.27\end{array}$ & $\begin{array}{l}\text { Toxicities noted } \\
\text { in all group; no } \\
\text { differences }\end{array}$ & $\begin{array}{l}\text { SRS alone; } \\
\text { chemotherapy } \\
\text { controlled }\end{array}$ \\
\hline $\begin{array}{l}\text { Diao et } \\
\text { al. } \\
\text { (2018) }\end{array}$ & NR & NR & NR & NR & NR \\
\hline $\begin{array}{l}\text { Diao et } \\
\text { al. } \\
\text { (2018) }\end{array}$ & NR & NR & NR & NR & NR \\
\hline $\begin{array}{l}\text { Yusuf } \\
\text { et al. } \\
(2017)\end{array}$ & NR & NR & NR & NR & NR \\
\hline
\end{tabular}

\section{TABLE 3: Studies considering treatment efficacy of SRS and targeted therapy}

SRS, stereotactic radiosurgery; NR, not reported in study outcomes; BRAFi, BRAF inhibitor; MEKi, MEK inhibitor

${ }^{\star}$ Converted from failure to control; *^Chemotherapy controlled

$[7-11]$ 


\section{Cureus}

\begin{tabular}{|c|c|c|}
\hline Study & Outcome & Reason for Exclusion \\
\hline Kotecha et al., (2017) & Improved LC, OS in concurrent SRS & High number (>30\%) WBRT \\
\hline $\begin{array}{l}\text { Mastorakos et al., } \\
\text { (2019) }\end{array}$ & Improved OS initiating inhibitor after SRS & No timing details, no HRs/statistics provided \\
\hline Wolf et al., (2016) & $\begin{array}{l}\text { Improved OS in concurrent or after SRS } \\
\text { strategies }\end{array}$ & No timing detalls, no HRS/statistics provided \\
\hline Xu et al., (2017) & Improved LC with any BRAFi + SRS & $\begin{array}{l}\text { Small sample size, heterogeneous timing. No HRs/statistics } \\
\text { provided }\end{array}$ \\
\hline Hecht et al., (2018) & Improved OS in an interrupted therapy group & High number (>30\%) WBRT \\
\hline \multicolumn{3}{|c|}{ TABLE 4: Excluded studies considering SRS + BRAFi/MEKi therapies } \\
\hline$[27-30,33]$ & & \\
\hline
\end{tabular}

\section{Conclusions}

Five studies in the literature explore concurrent timing of stereotactic radiosurgery and immunotherapy or targeted therapies for the treatment of intracranial metastatic melanomas. Additional temporally specific studies are needed, but more vital is the need for well-designed prospective trials, several of which are under way [NCT02085070; NCT02374242; NCT02460068; NCT02320058]. Future studies should report outcomes based on a four-week window (concurrent SRS) or a three-month window (peri-SRS).

\section{Additional Information}

\section{Disclosures}

Conflicts of interest: In compliance with the ICMJE uniform disclosure form, all authors declare the following: Payment/services info: All authors have declared that no financial support was received from any organization for the submitted work. Financial relationships: All authors have declared that they have no financial relationships at present or within the previous three years with any organizations that might have an interest in the submitted work. Other relationships: All authors have declared that there are no other relationships or activities that could appear to have influenced the submitted work.

\section{Acknowledgements}

We thank Kristin Kraus, MSc, for editorial assistance.

\section{References}

1. Davies MA, Liu P, McIntyre S, et al.: Prognostic factors for survival in melanoma patients with brain metastases. Cancer. 2011, 117:1687-1696. 10.1002/cncr.25634

2. Peuvrel L, Saint-Jean M, Quéreux G, Brocard A, Khammari A, Knol A, Dréno B: Incidence and characteristics of melanoma brain metastases developing during treatment with vemurafenib. J Neurooncol. 2014, 120:147154. 10.1007/s11060-014-1533-z

3. Flaherty KT, Infante JR, Daud A, et al.: Combined BRAF and MEK inhibition in melanoma with BRAF V600 mutations. N Engl J Med. 2012, 367:1694-1703. 10.1056/NEJMoa1210093

4. Hodi FS, O'day SJ, McDermott DF, et al.: Improved survival with ipilimumab in patients with metastatic melanoma. N Engl J Med. 2010, 363:711-723. 10.1056/NEJMoa1003466

5. Robert C, Thomas L, Bondarenko I, et al.: Ipilimumab plus dacarbazine for previously untreated metastatic melanoma. N Engl J Med. 2011, 364:2517-2526. 10.1056/NEJMoa1104621

6. Dovedi $\mathrm{S}$, Illidge $\mathrm{T}$ : The antitumor immune response generated by fractionated radiation therapy may be limited by tumor cell adaptive resistance and can be circumvented by PD-L1 blockade. Oncoimmunology. 2015, 4:1016709. 10.1080/2162402X.2015.1016709

7. Acharya S, Mahmood M, Mullen D, et al.: Distant intracranial failure in melanoma brain metastases treated with stereotactic radiosurgery in the era of immunotherapy and targeted agents. Adv Radiat Oncol. 2017, 2:572-580. 10.1016/j.adro.2017.07.003

8. Ahmed KA, Abuodeh YA, Echevarria MI, et al.: Clinical outcomes of melanoma brain metastases treated with stereotactic radiosurgery and anti-PD-1 therapy, anti-CTLA-4 therapy, BRAF/MEK inhibitors, BRAF inhibitor, or conventional chemotherapy. Ann Oncol. 2016, 27:2288-2294. 10.1093/annonc/mdw417

9. Diao K, Bian SX, Routman DM, et al.: Combination ipilimumab and radiosurgery for brain metastases: tumor, edema, and adverse radiation effects. J Neurosurg. 2018, 129:1397-1406. 10.3171/2017.7.JNS171286

10. Diao K, Bian SX, Routman DM, et al.: Stereotactic radiosurgery and ipilimumab for patients with melanoma 
brain metastases: clinical outcomes and toxicity. J Neurooncol. 2018, 139:421-429. 10.1007/s11060-0182880-y

11. Yusuf MB, Amsbaugh MJ, Burton E, Chesney J, Woo S: Peri-SRS administration of immune checkpoint therapy for melanoma metastatic to the brain: investigating efficacy and the effects of relative treatment timing on lesion response. World Neurosurg. 2017, 100:632-640. 10.1016/j.wneu.2017.01.101

12. Demaria S, Ng B, Devitt ML, Babb JS, Kawashima N, Liebes L, Formenti SC: Ionizing radiation inhibition of distant untreated tumors (abscopal effect) is immune mediated. Int J Radiat Oncol Biol Physics. 2004, 58:862-870. 10.1016/j.ijrobp.2003.09.012

13. Dewan MZ, Galloway AE, Kawashima N, Dewyngaert JK, Babb JS, Formenti SC, Demaria S: Fractionated but not single-dose radiotherapy induces an immune-mediated abscopal effect when combined with anti-CTLA4 antibody. Clin Cancer Res. 2009, 15:5379-5388. 10.1158/1078-0432.CCR-09-0265

14. Mole R: Whole body irradiation-radiobiology or medicine?. Br J Radiol. 1953, 26:234-241. 10.1259/00071285-26-305-234

15. Demaria S, Kawashima N, Yang AM, Devitt ML, Babb JS, Allison JP, Formenti SC: Immune-mediated inhibition of metastases after treatment with local radiation and CTLA-4 blockade in a mouse model of breast cancer. Clin Cancer Res. 2005, 11:728-734.

16. Kaminski JM, Shinohara E, Summers JB, Niermann KJ, Morimoto A, Brousal J: The controversial abscopal effect. Cancer Treat Rev. 2005, 31:159-172. 10.1016/j.ctrv.2005.03.004

17. Kiess AP, Wolchok JD, Barker CA, et al.: Stereotactic radiosurgery for melanoma brain metastases in patients receiving ipilimumab: Safety profile and efficacy of combined treatment. Int J Radiat Oncol Biol Physics. 2015, 92:368-375. 10.1016/j.ijrobp.2015.01.004

18. Burnette BC, Liang H, Lee $\mathrm{Y}$, et al.: The efficacy of radiotherapy relies upon induction of type I interferondependent innate and adaptive immunity. Cancer Res. 2011, 71:2488-2496. 10.1158/0008-5472.CAN-102820

19. Brocks D, Schmidt CR, Daskalakis M, et al.: DNMT and HDAC inhibitors induce cryptic transcription start sites. Nat Genet. 2017, 49:1052. 10.1038/ng.3889

20. Cañadas I, Thummalapalli R, Kim JW, et al.: Tumor innate immunity primed by specific interferonstimulated endogenous retroviruses. Nat Med. 2018, 24:1143-1150. 10.1038/s41591-018-0116-5

21. Chiappinelli KB, Strissel PL, Desrichard A, et al.: Inhibiting DNA methylation causes an interferon response in cancer via dsRNA including endogenous retroviruses. Cell. 2015, 162:974-986. 10.1016/j.cell.2015.07.011

22. Chapman PB, Hauschild A, Robert C, et al.: Improved survival with vemurafenib in melanoma with BRAF V600E mutation. N Engl J Med. 2011, 364:2507-2516. 10.1056/NEJMoa1103782

23. Chapman PB, Solit DB, Rosen N: Combination of RAF and MEK inhibition for the treatment of BRAFmutated melanoma: feedback is not encouraged. Cancer Cell. 2014, 26:603-604. 10.1016/j.ccell.2014.10.017

24. Hauschild A, Grob J-J, Demidov LV, et al.: Dabrafenib in BRAF-mutated metastatic melanoma: a multicentre, open-label, phase 3 randomised controlled trial. Lancet. 2012, 380:358-365. 10.1016/S01406736(12)60868-X

25. Long G, Flaherty K, Stroyakovskiy D, et al.: Dabrafenib plus trametinib versus dabrafenib monotherapy in patients with metastatic BRAF V600E/K-mutant melanoma: long-term survival and safety analysis of a phase 3 study. Ann Oncol. 2017, 28:1631-1639. 10.1093/annonc/mdx176

26. Wolf A, Zia S, Verma R, et al.: Impact on overall survival of the combination of BRAF inhibitors and stereotactic radiosurgery in patients with melanoma brain metastases. J Neurooncol. 2016, 127:607-615. 10.1007/s11060-016-2072-6

27. Kotecha R, Miller JA, Venur VA, et al.: Melanoma brain metastasis: the impact of stereotactic radiosurgery, BRAF mutational status, and targeted and/or immune-based therapies on treatment outcome. J Neurosurg. 2017: 1-10. 10.3171/2017.1.JNS162797

28. Mastorakos $\mathrm{P}, \mathrm{Xu} \mathrm{Z}, \mathrm{Yu}$ J, et al.: BRAF V600 mutation and BRAF kinase inhibitors in conjunction with stereotactic radiosurgery for intracranial melanoma metastases: a multicenter retrospective study. Neurosurgery. 2019, 84:868-880. 10.1093/neuros/nyy203

29. Xu Z, Lee C-C, Ramesh A, et al.: BRAF V600E mutation and BRAF kinase inhibitors in conjunction with stereotactic radiosurgery for intracranial melanoma metastases. J Neurosurg. 2017, 126:726-734. 10.3171/2016.2.JNS1633

30. Hecht M, Meier F, Zimmer L, et al.: Clinical outcome of concomitant vs interrupted BRAF inhibitor therapy during radiotherapy in melanoma patients. Br J Cancer. 2018, 118:785-792. 10.1038/bjc.2017.489

31. Hecht M, Zimmer L, Loquai C, et al.: Radiosensitization by BRAF inhibitor therapy-mechanism and frequency of toxicity in melanoma patients. Ann Oncol. 2015, 26:1238-1244. 10.1093/annonc/mdv139

32. Kroeze SGC, Fritz C, Hoyer M, et al.: Toxicity of concurrent stereotactic radiotherapy and targeted therapy or immunotherapy: A systematic review. Cancer Treat Rev. 2017, 53:25-37. 10.1016/j.ctrv.2016.11.013

33. Wolf A, Zia S, Verma R, et al.: Impact on overall survival of the combination of BRAF inhibitors and stereotactic radiosurgery in patients with melanoma brain metastases. J Neurooncol. 2016, 127:607-615. 10.1007/s11060-016-2072-6 